\title{
Numerical Simulation of a Centrifugal Compressor
}

\author{
S.M.Swamy*, V.Panndurangadu** and R. Rama Chandra*** \\ * Assistant Professor, Department of Mechanical Engineering, GNITS, Shaikpet, Hyderabad-500104, India. \\ ** Professor, Department of Mechanical Engineering, JNTUA, Ananthapuramu -515002, A.P, India. \\ *** Professor \& Principal, Department of Mechanical Engineering SKD Engineering College, Gooty, A.P.
}

\begin{abstract}
In this paper, the tip clearance effects on flow field of a low speed centrifugal compressor without and with partial shroud (PS) fitted on to the rotor blade tip at three values of tip clearance, viz. $\tau=2.2 \%, 5.1 \%$ and $7.9 \%$ of rotor blade height at the exit at three flow coefficients, namely, $\phi=0.18,0.28$, and 0.34 , was studied. Based on the theory of computational fluid dynamics (CFD), performance curves and parameter distributions of the compressor were obtained from the 3-D numerical simulation by using ANSYS CFX 15.0. The centrifugal compressor in aerodynamic requirement is that edge velocities along the impeller channel passage surfaces like hub, shroud, pressure and suction surfaces vary smoothly without sudden decelerations, which cause flow separation leading to losses. Using the periodic boundaries and defined flow conditions at inflow / exit flow and blade rotations, the turbulent viscous flow between blade channels are computed. The efficiency related parameters using average quantities, besides flow pattern in terms of velocities, streamlines and pressure distribution on blade surfaces are graphically interpreted. An attempt is also made to study the influence of pressure loads on structural deformations in the chosen blade profile. This paper highlights aero-mechanical features of centrifugal impeller obtained from several numerical simulations, which are expected to provide a sound basis for further investigations.
\end{abstract}

Keywords: Centrifugal compressor, Flow coefficient, Partial shroud (PS) attached to the rotor blade tip, Streamlines, passage wake.

\section{INTRODUCTION}

The centrifugal compressors have an extensive variety of applications particularly for power plants for small aircraft and helicopters, in process industries, compression of gases and vapours, because they can provide high-pressure ratios and large operating ranges with relatively high efficiencies. Centrifugal compressors are used primarily for their suitability for handling small volume flows, but other advantages include a shorter length than an equivalent axial flow compressor, less susceptibility to loss of performance by buildup of deposits on the blade surfaces and their suitability to operate over a wide range of mass flow. The gas turbine engines powering the most passenger and military aircrafts are also called jet engines. Tip clearance in centrifugal compressor causes the leakage of high pressure fluid from pressure surface to suction surface of the impeller blade, making the flow field highly complex and effecting the performance. In centrifugal compressor due to the low efficiency and thrust to weight ratio, they are limited to short range applications. However, the inability for spark ignition engines to work at high altitude warrants further development to improve the efficiency of these miniature gas turbines. Hayami (1997) has found from his experiments that axial movement of the casing has better efficiency over the movement of casing in radial and axial directions. Radial movement of casing increases clearance at inducer, which reduces the operating range. The tip clearance studies are conducted to understand the flow behavior in order to minimise the effect of tip clearance. Pampreen (1973), Mashimo et al. (1979), Sitaram and Pandey (1990) have conducted experimental studies and suggested that by reducing the tip clearance gap size, the tip clearance effect can be minimised.

\section{MODELING OF COMPRESSOR BLADES}

The major geometrical details of the impeller are total pressure rise, $\Delta \mathrm{P}: 300 \mathrm{~mm}$ WG, Speed of rotation, N: $2000 \mathrm{rpm}$, Impeller inlet diameter at inducer tip, $\mathrm{d}_{1 \mathrm{t}} ; 300 \mathrm{~mm}$, Impeller inlet diameter at inducer hub, $\mathrm{d}_{1 \mathrm{~h}} ; 160 \mathrm{~mm}$, Impeller outer diameter, $\mathrm{d}_{2}: 500 \mathrm{~mm}$, Blade angle at exit: (a) At hub: $75^{\circ}$, (b) At mean section: $90^{\circ}$, (c) At shroud: $105^{\circ}$, Blade angle at inlet inducer hub, $\beta_{1 \mathrm{~h}}$ : $53^{\circ}$, Blade angle at inducer tip, $\beta_{1 \mathrm{t}}: 35^{\circ}$, Blade width at the exit, b: $34.7 \mathrm{~mm}$, No. of blades of the impeller, $\mathrm{N}_{\mathrm{b}}$ : 16. All the angles are with respect to the tangential direction. 


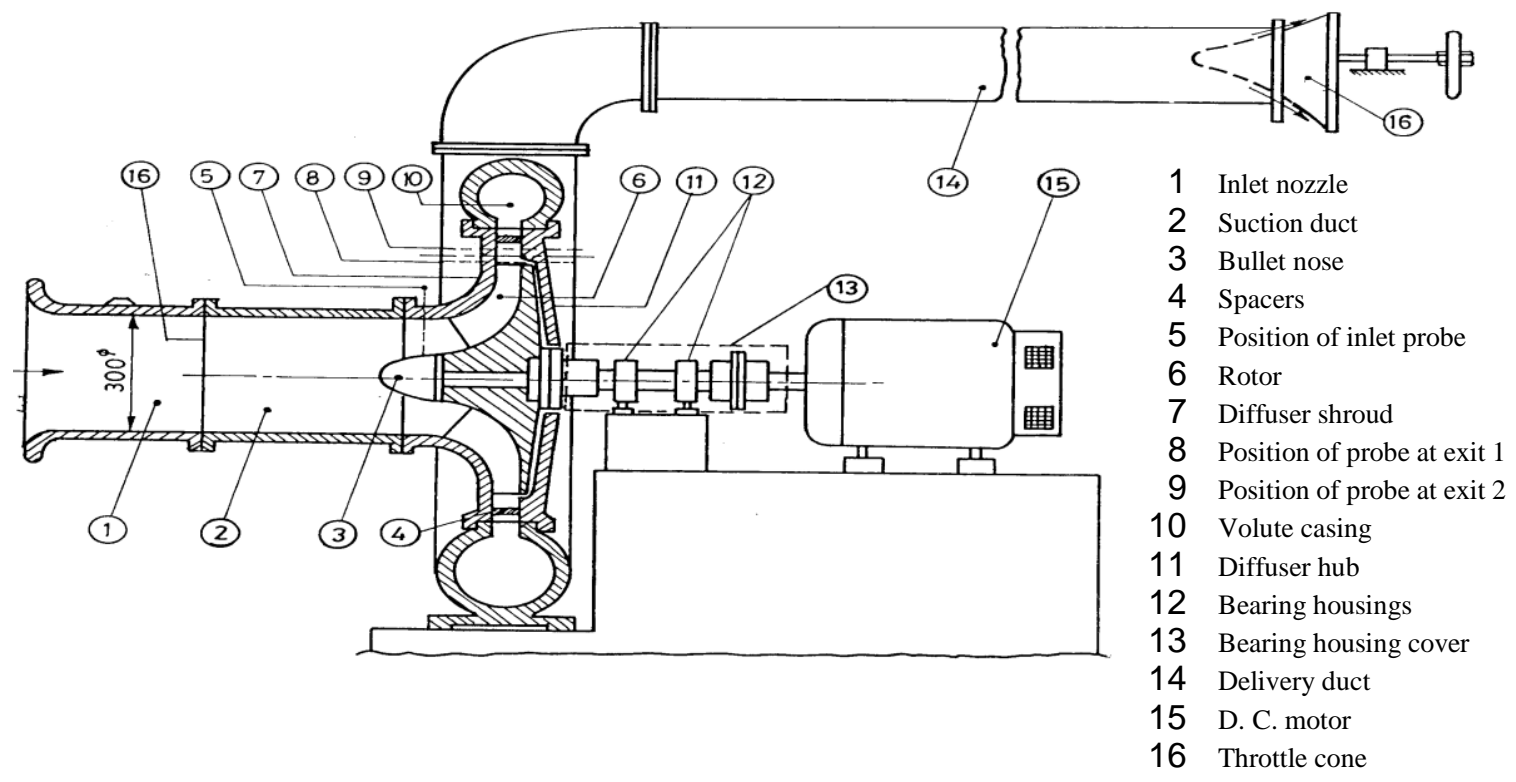

Fig. 1 Schematic diagram of centrifugal compressor setup

Schematic diagram of centrifugal compressor setup as shown in fig.1. The geometric model used for the computational simulation of Centrifugal impeller with above specifications with $3 \mathrm{~mm}$ thickness throughout the blade for generation of three dimensional blades was done as shown in Fig. 2. A single passage of the impeller with inlet at $50 \mathrm{~mm}$ ahead of the impeller and outlet at a distance of $35 \mathrm{~mm}$ downstream of impeller is shown in Fig. 3. Casing is designed with $0.7 \mathrm{~mm}$ clearance throughout the blade height. Three tip clearances of $2.2 \%, 5.1 \%$ and $7.9 \%$ of trailing edge blade height are obtained by moving the casing axially. ANSYS CFX 15.0 version software is used for obtaining the solution and standard $\mathrm{k}-\varepsilon$ turbulence model is used for the closure. The computational domain is kept at $50 \mathrm{~mm}$ ahead of the eye inlet of the impeller in order to ensure that the inlet boundary conditions are not affected by the back pressure of impeller blade. The blade gen layout with construction features related to wrapper angle, number of blades, angles distribution and blade thickness for generation of three dimensional blades was done in order to carry out structural analysis of the blade surfaces the software provided export options to generate computational mesh accounting blade thickness is also explored in static structural analysis package. The blade profiles generated in above software module are imported to template based multi-block structured computational mesh software module called turbo grid. The information related to machine data, profile curves, several inputs required for width factor periodicity production, topology freezing for generation of geometric representation in terms of surface mesh generation with master and slave control points being generated. default orientation of master and slave control points on the blade surfaces are to be moved to obtain better orthogonally between mesh points. After these operations three dimensional computational mesh generation process for compressor blade with boundary patches, periodic surfaces are created.
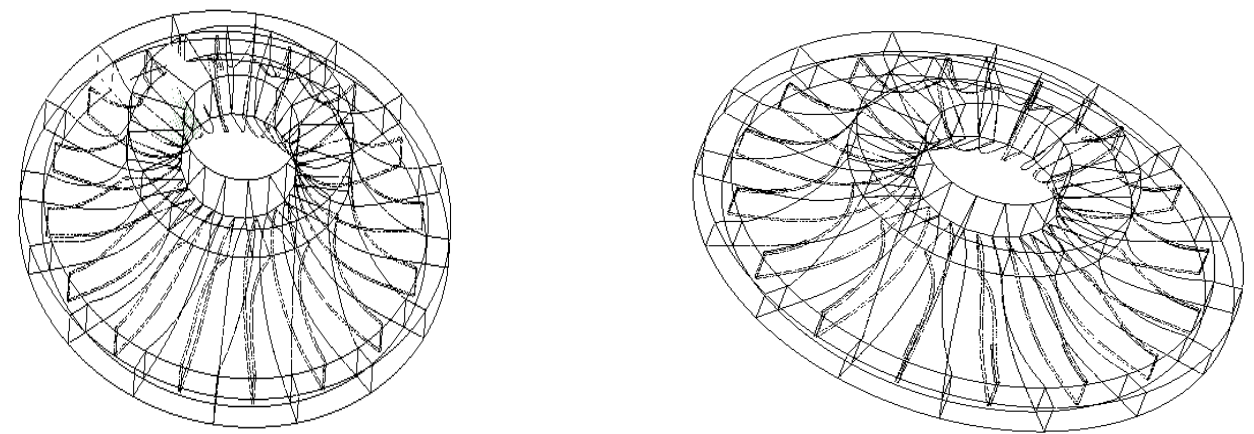

Fig. 2 Centrifugal compressor (without and with PS) 

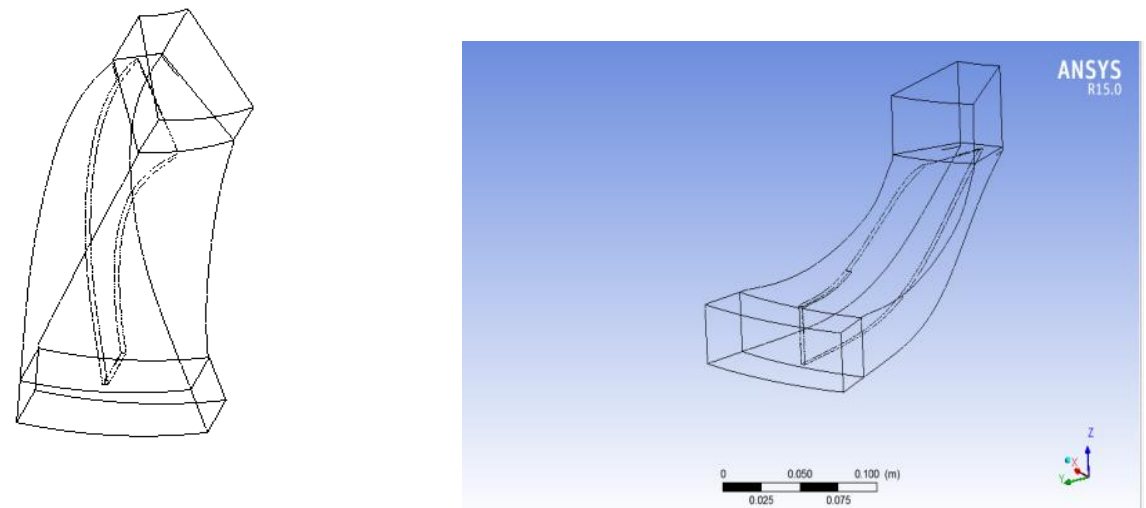

Fig. 3 Computational domain of single passage (without and with PS)

\section{COMPUTATIONAL MESH FOR STRUCTURAL ANALYSIS}

Most fluid flow simulation technology uses completely different meshing, loading and solving methods compared to structural simulation. And this is where the true power of Workbench 15.0 comes into play. When the user is connecting up the dialogs within the schematic layouts, many of these are handled automatically, simply by dragging a connection between the outputs of one analysis system and the inputs of another. When and if there's mapping to be done, this is handled in a very simple dialog that makes the process simple, rather than the often-tortuous methods traditionally associated with this type of work. There are no geometry creation tools in the Mechanical application and so geometry must be attached to the Mechanical application. The geometry from either of the CAD Systems and Blade Gen Model can be connected to simulation model before setup option is activated. The next step is to generate the meshing solid body for which several methods are provided in ANSYS Workbench 15.0 these tools have the benefit of being highly automated along with having a moderate to high degree of user control. Upon startup of the meshing application meshing options panel displays, which allows you to quickly and easily set your meshing preferences based on the physics you are preparing to solve. For solid bodies, the software uses meshing techniques based on algorithms and element shape. One of the technique extensively used are called patch independent meshing for the part using tetrahedral element. The default value selects meshing control parameters and provides a successful automated mesh. By default, the application attempts to use auto sweeping for solid models and quadrilateral element generation for surface body models. If a model is not sweepable, the Patch Conforming mesher under tetrahedrons is used. Then one needs to change the default values suitably in the Mesh option panel. Patch independent mesh are defined by max element size and number of elements. The default value is calculated from the diagonal length of the bounding box which is based on the initial seed settings of mesh object folder. When curvature and proximity refinement options enabled, the mesh automatically refined based on geometry. This will result in larger elements on flat planar faces and smaller elements in areas of high curvature or within small gaps. However projection to geometry and smoothing may push the size even smaller for some of the elements as observed in blade leading edges.

\section{FLOW SIMULATION IN CENTRIFUGAL COMPRESSOR BLADE}

To verify the performance of centrifugal compressor using computational fluid dynamics commercial codes ANSYS CFX 15.0. We modeled the geometries with and without partial shroud using CATIA modeling software procedure. We imported the airfoil sections at certain heights then made the surface using multi-section tool in GSD module of CATIA software. By closing upper and bottom surface we get the solid section of an one blade we have to add the partial shroud at tip of the blade at its trailing edge with the thickness of given height and $50 \mathrm{~mm}$ in length along the blade and $2 \mathrm{~mm}$ width more than actual blade thickness from its pressure side of airfoil. Next, extraction of fluid domain total number of blades for this particular centrifugal compressor are 16 so it's possible to simulate only one periodic section of fluid to interpolate the results for other remaining sections of fluid domain. This extraction is also based on equalized divisions of cylindrical fluid domain. We have extracted fluid domain in CATIA software itself. Meshing is the next step, in the meshing nodes on the higher and lower periodic surfaces we should have equal number of nodes. Match control option is used for that. Name selection is important for each surface of non-conformable meshing in 
rotating bodies (i.e. with sliding contact notion). CFX can detect automatically the respective surfaces names for respective parts of compressor. For each surface given a meaning full name as shown in Fig.4 (a), where S1 and S2 representing stationary inflow and outflow domains respectively. $\mathrm{R} 1$ is rotating domain which containing blade surfaces (including partial shroud) inside it. As standard notations are used to represent the shroud, hub, periodic surfaces, blade surfaces, and inflow outflow surfaces.

The quality of meshing and number of elements is very important concern for CFD simulation this can be seen in below table. Quality of mashing is measured usually by skewness present in meshing. It's a ratio of smallest angle between edges of element present is actual meshing by angle between edges in standard element (angle for standard elements are $60^{\circ}$ for triangular mesh and $90^{\circ}$ for rectangular mesh). In other words it's showing fraction of deviation of element shape from standard element shape. In below case maximum skewness is 0.7977 which is acceptable this value should be less than 0.9. Number of elements are 2310116. This much of elements are require testing mesh independency.

\section{Project \\ 官.... Model (B3) \\ †..... Geometry \\ 我.... Coordinate Systems \\ †...... Connections \\ Match Control \\ Match Control 2 \\ 1. Match Control 3 \\ 17 Match Control 4 \\ 17 Match Control 5 \\ 19. Face Sizing \\ ๑..... Named Selections \\ Shroud_Inblock \\ (1) Hub_Inblock \\ Inlet_Inblock \\ Pi Otflow_Inblock \\ Inflow_R1 \\ Periodic-1_Inblock \\ II Periodic-2_Inblock \\ T1 Periodic-1_R1 \\ PI Periodic-2_R1 \\ Shroud_R1 \\ . Tub_R1 \\ II Outflow_R1 \\ Phroud_S2 \\ Q1 Inflow_S2 \\ (1) Periodic-1_S2 \\ I Outflow_S2 \\ Pub_S2 \\ Pil Periodic-2_S2 \\ it Blade_R1}

a)

\begin{tabular}{|l|l|}
\hline Statistics \\
\hline$\square$ Nodes & 445604 \\
\hline$\square$ Elements & 2310116 \\
\hline Mesh Metric & Skewness \\
\hline$\square$ Min & $7.666276246443 \mathrm{E}-05$ \\
\hline$\square$ Max & 0.797751114670892 \\
\hline$\square$ Average & 0.233106168038319 \\
\hline$\square$ Ctandard naviation & n 1 12661122A2nn1 \\
\hline
\end{tabular}

b)

Fig.4 a) Meshing Details with Name Selection b) mesh statistics

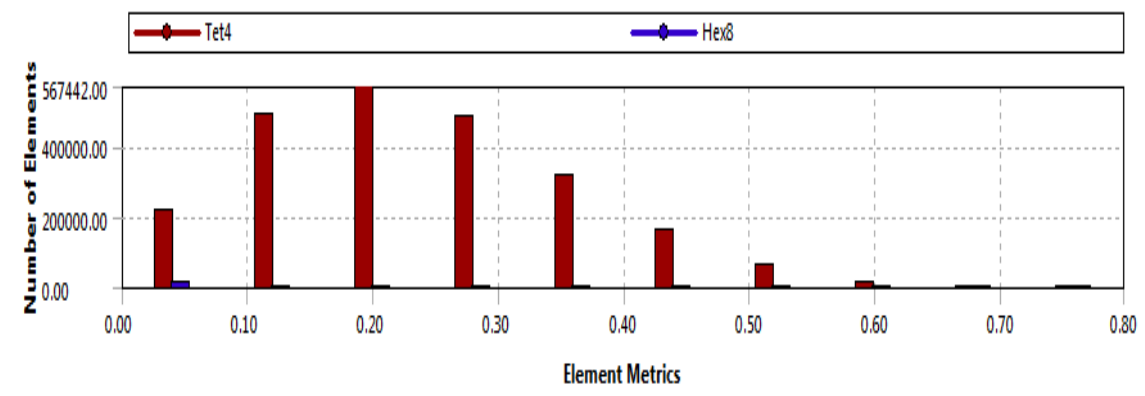

Fig.5. Frequency of distribution of skewness. 
In CFX we have to use turbo mode for this problem statement, with respective to this stationary, rotating components are defined. As the density going to vary (compressible fluid) with pressure fluid properties air kept as ideal gas. Reference pressure is $1 \mathrm{~atm}$. Heat transfer is calculated by total energy because increase in density causes to increase in temperature and because of rotational energy it will try to change the heat transfer rate, in total energy methods only all this effects will get considered. Shear Stress Transport Turbulence model was used because of near wall treatment is required and presence of ad- versed pressure gradient is present. Inlet and outlet boundaries are pressure inlet with $0 \mathrm{~Pa}$ gauge pressure and mass flow $0.087 \mathrm{Kg} / \mathrm{s}$ at outlet respectively. Rotating domain interface used as frozen rotor. Stage for interfaces used for steady state simulations, rotational interaction between reference frames is not accounted for - i.e. the stationary domain "sees" the rotating components at a fixed position. Physical Time scaling value was observed to be taken as $10^{-3} \mathrm{~s}$. Accuracy criteria of RMS solution was decreased to $10^{-5}$ for better accuracy.
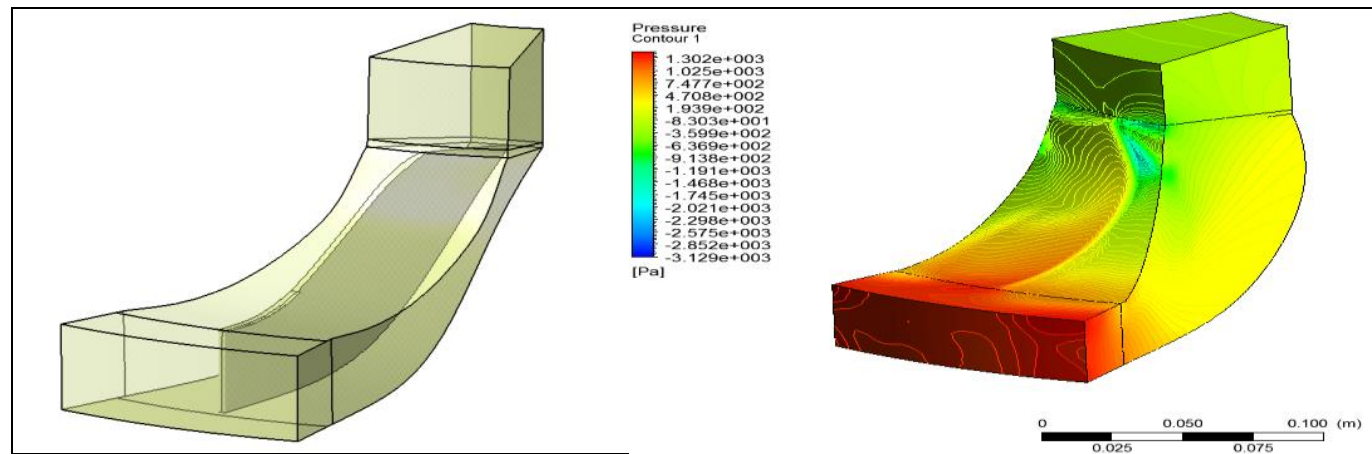

Fig. 6 Extracted Fluid Domain Fig.7 Pressure at outlet, casing, periodic surface and inlet (Clearance 2.2\% without PS)

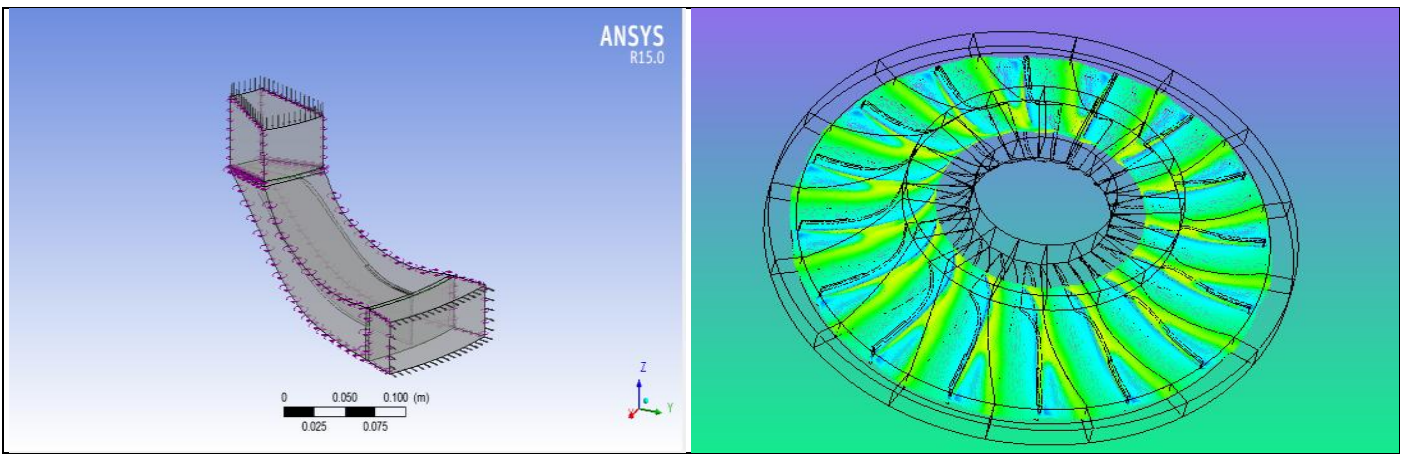

Fig. 8 Three dimensional mesh for single compressible blade row and streamlines from the inlet of impellor around the blades

\section{RESULTS AND DISCUSSIONS}

The impellor blades are rotating with 2000 rpm is subjected to mass flow $0.087 \mathrm{~kg} / \mathrm{sec}$ with the working pressure is of order $1 \mathrm{~atm}$. The pressure loading over stream wise directions for the span of $70 \%$ is shown in the Figure. The pressure profiles on the surfaces near hub mean and tip region provides details of boundary layer separation with steep adverse pressure gradients [6]. Pressure drops rapidly with the increase in mass flow rate and this will lead to choking at a certain point. On the other hand, pressure also drops accordingly as the mass flow rate is reduced from that optimum point and the stage will quickly start to surge.

\section{Velocity Vectors at Outlet}

A velocity vector at the exit of the impeller is shown in fig. 9. The fluid flows with PS on tip of the blade velocities are high on suction surface than pressure surface because of blade curvature. With increase in tip clearance, the velocity on both pressure side and suction side is decreasing. For $2.2 \%$ and $5.1 \%$ clearance, high velocity of the fluid above the blade from pressure side to suction side through tip clearance is clearly seen. 


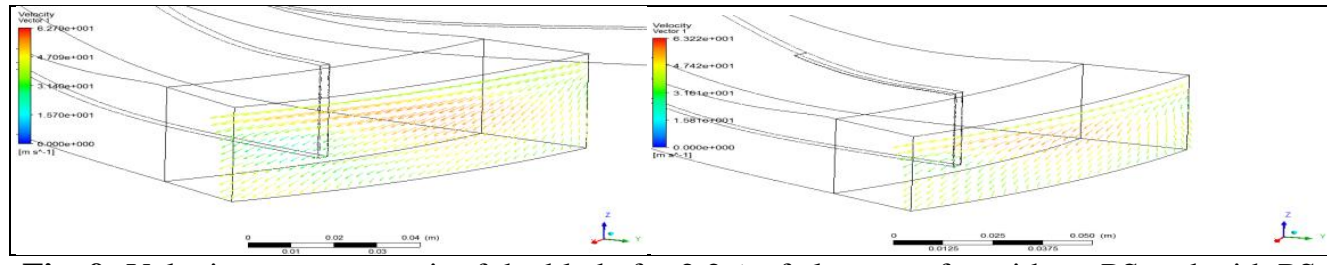

Fig. 9: Velocity vectors at exit of the blade for $2.2 \%$ of clearance for without PS and with PS

\section{Static Pressure Contours at Span 0.7}

Pressure contours in blade to blade view, at span 0.7 is shown in figure 10 . The contours show gradual pressure rise from inlet to outlet of the compressor due to dynamic action of the rotating impeller. Gradual increase of static pressure from inlet to outlet is clearly observed at all tip clearances. With PS on tip of the blade, low pressure change is observed. But without PS on tip of the blade, the pressure at outlet is reduced. High pressure on pressure side of the blade and low pressure on suction side of the blade are observed at all tip clearances. With increase in tip clearance, reduction in pressure on both pressure side and suction side is found.

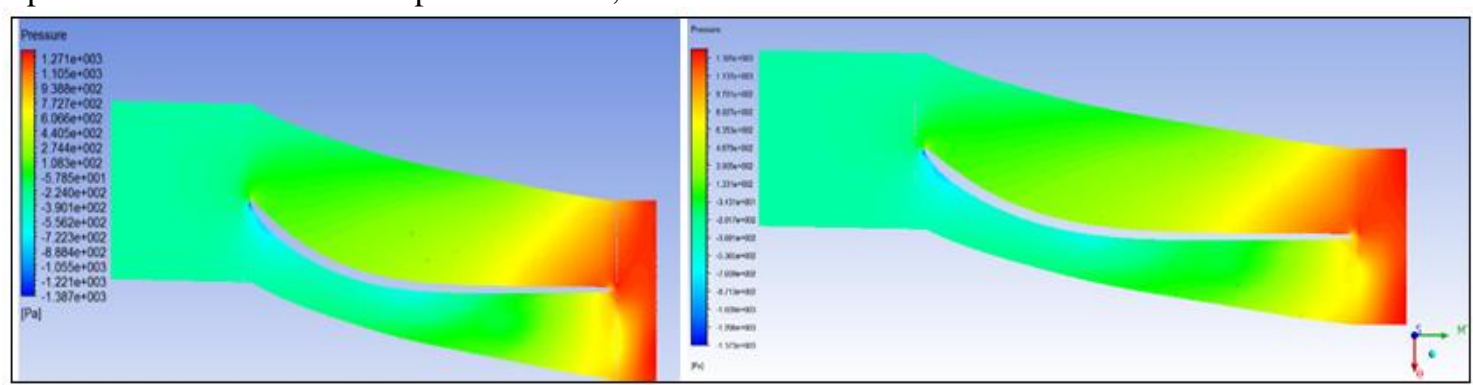

Fig.10: Static pressure contours for $2.2 \%$ clearance at mid span without PS and with PS

\section{Velocity Contours at Span 0.7}

Velocity contours in blade to blade view, at span 0.7 is shown in figure 11. The contours show low velocity region on suction side of the blade. With PS on tip of the blade, the low velocity region is reducing and also velocity improvement is observed.
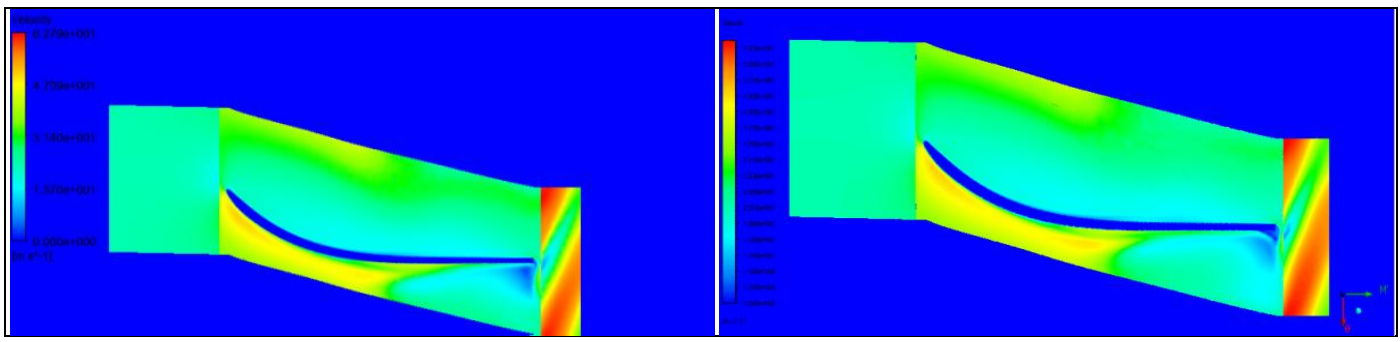

Fig. 11: Velocity contours for $2.2 \%$ clearance for without PS and with PS

Outlet to inlet pressure ratio at different flow coefficients is increase in pressure ratio with PS on tip of the blade is observed at all flow coefficients. But without PS on tip of the blade, reduction in pressure ratio is observed at all flow coefficients because of more fluid leakage through the basic configuration.

\section{Blade loading curves:}

Blade loading curves at design flow coefficient 0.28 for $2.2 \%$ tip clearance at without PS and with PS were shown in fig. 12. Fluid flow without PS and with PS on tip of the blade, significant change in pressure on suction side is observed. Low static pressure on suction and high pressure on pressure side of the blade is observed. With increase in tip clearance, static pressure on both pressure side and suction side are reducing. 

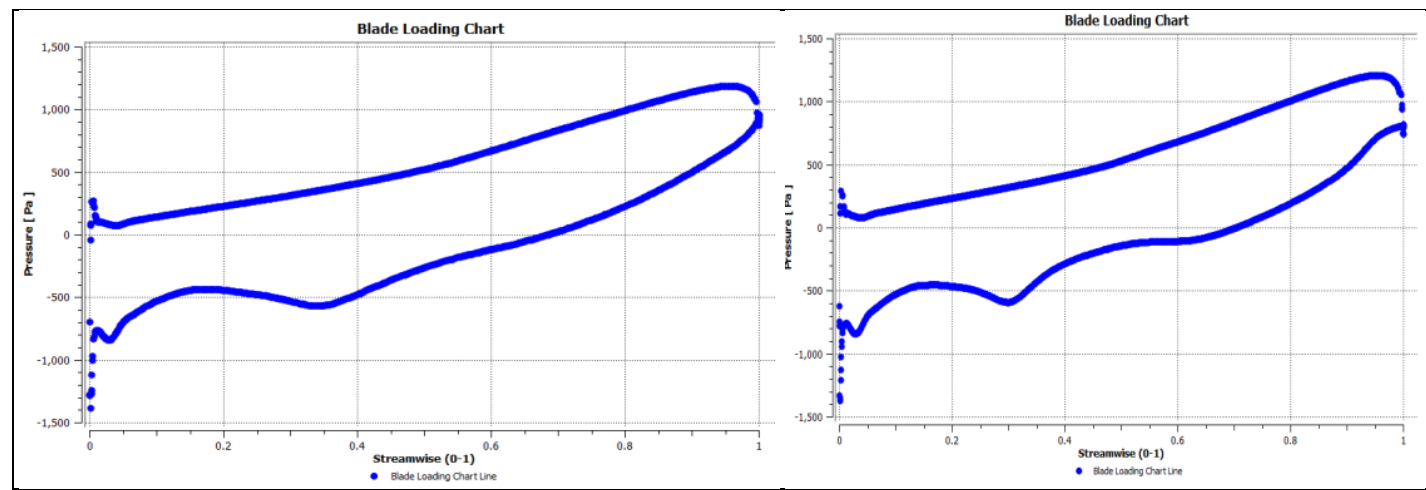

Fig.12: Blade loading for $2.2 \%$ clearance at without and with partial shroud

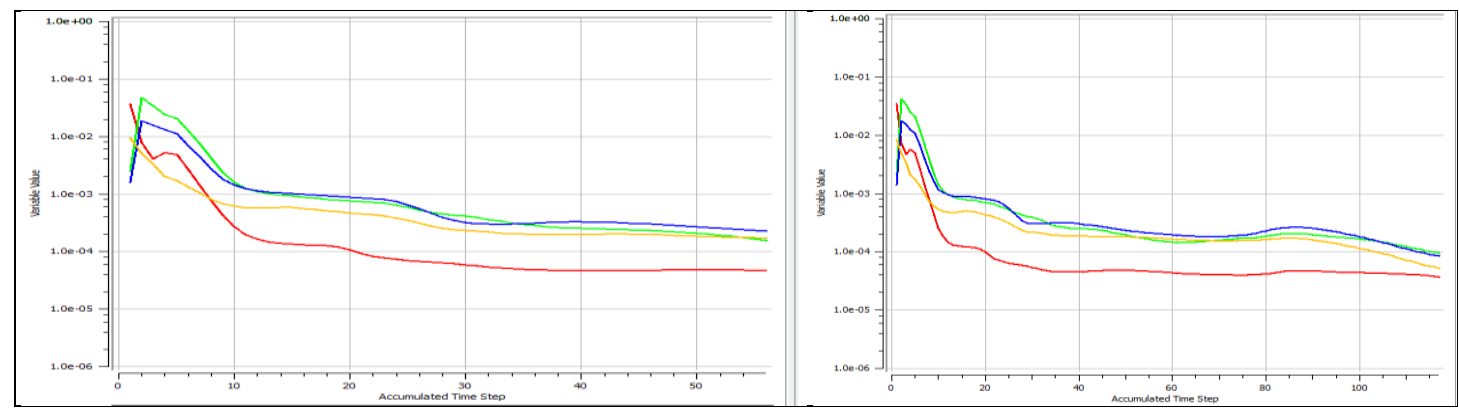

Fig.13: Momentum, Heat transfer and Mass converged residual plot. (Without and with PS)
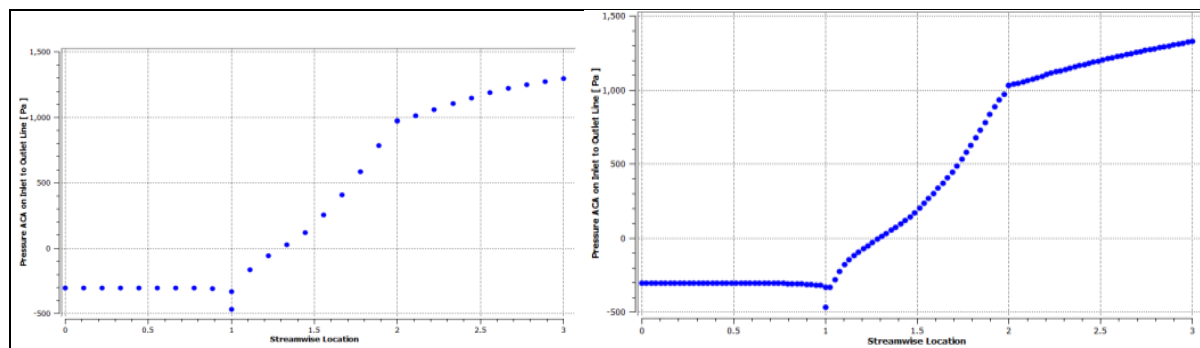

Fig.14: Static pressure distribution from inlet to outlet over stream wise (without and with PS) for $2.2 \%$ of clearance.

\section{CONCLUSIONS}

Tip clearance effects on flow field of a centrifugal compressor without and with partial shroud (PS) attached to the rotor blade tip at three values of tip clearance, viz. $\tau=2.2 \%, 5.1 \%$ and $7.9 \%$ of rotor blade height at the exit at three flow coefficients, namely, $\phi=0.18,0.28$ and 0.34 , is analysed computationally using structured multi block grid with fine grid in the tip clearance region. Velocity contours in blade to blade view; at span 0.7 is low velocity region on suction side of the blade. With PS on tip of the blade, the low velocity region is reducing then velocity improved. Blade loading charts at design flow coefficient 0.28 for $2.2 \%$ tip clearance for fluid flow without PS and with PS on tip of the blade, significant change in pressure on suction side is observed. Low static pressure on suction and high pressure on pressure side of the blade is observed. With increase in tip clearance, static pressure on both pressure side and suction side are reducing. It is observed that maximum stress is taking place at the hub surface trailing edge and minimum stress is noticed on shroud surface when pressure load obtained from CFD solution is specified on the blade surfaces. As mass flow varies at constant rotational speeds the magnitude of maxima and minima of stresses decrease. Similarly, the total deformation also decreases at constant speed at different mass flow rates. The pressure from inlet to the outlet of the compressor is increasing gradually along the stream wise direction due to the dynamic head developed by the rotating impeller. A drop in static pressure near streamwise direction is observed for two cases due to the acceleration of the flow in to the eye of the impeller.

The pressure contours show that there is a shifting of low momentum fluid from suction surface to pressure surface in tip clearance region due to tip leakage flow. Wake region near suction side shroud corner is observed. Wake region 
growth and movement towards the mid pitch with increase in clearance is observed.

\section{REFERENCES}

[1]. Fowler, H. S., (1971) Aerodynamics Tests on a Centrifugal Fan Impeller Model with Swept Back Blades, N.R.C. Report No. 12020.

[2]. Senoo, Y. And M. Ishida, (1975) Behaviour of Severely Asymmetric Flow in a Vaneless Diffuser, Trans. ASME, Jl. of Engg. for Power, 97, 375-382

[3]. Moore, J., (1973), "A Wake and an Eddy in a Rotating Radial-Flow Passage", Part 1: Experimental Observation", Part 2:"Flow Model", Trans. ASME Journal of Engineering for Power.pp.205-219.

[4]. Eckardt, D., (1976) Detailed Flow Investigations within a High Speed Centrifugal Compressor Impeller, Trans. ASME, Jl. of Fluids Engg. 98, 390-402.

[5]. Adler, D., (1980) Status of Centrifugal Internal Aerodynamics, Part II: Experiments and Influence of Viscosity, Trans. ASME, Jl. of Engineering for Power, 102, 738-746

[6]. Shivaprasad, B. G. and B. R. Ramaprian, (1977) Turbulence Measurements in Boundary Layers along Mildly Curved Surfaces, ASME Paper 77-WA/FE-8.

[7]. Krain, H. and D. Eckardt, (1978) The Flow Field in a High Speed Centrifugal Impeller-A Comparison of Experimental and Theoretical Results, First International Conference on Centrifugal Compressor Technology, IIT Madras.

[8]. Johnson, M.W., and Moore, J., (1980), "The development of wake flow in a centrifugal compressor" ASME Journal of Engineering for Power, Vol. 102, pp. 383-390.

[9]. Johnson, M. W. and J. Moore, (1983) Secondary Flow Mixing Losses in a Centrifugal Impeller, Trans. ASME, Jl. of Engg. for Power, 105, 24-32. 